OPEN ACCESS

Edited by:

Patrick J. Schuler,

Ulm University Medical Center,

Germany

Reviewed by:

Roberto Montalti,

Federico II University Hospital, Italy

Tommaso Maria Manzia,

University of Rome Tor Vergata, Italy

*Correspondence:

Maogen Chen maogen2000@163.com

Zhiyong Guo

rockyucsf1981@126.com

Xiaoshun $\mathrm{He}$

gdtrc@163.com

${ }^{\dagger}$ These authors have contributed equally to this work and share first authorship

Specialty section: This article was submitted to Surgical Oncology, a section of the journal

Frontiers in Oncology

Received: 10 September 2021 Accepted: 22 November 2021 Published: 13 December 2021

Citation:

Tang Y, Wang T, Ju W, Li F, Zhang Q, Chen Z, Gong J, Zhao Q, Wang $D$, Chen M, Guo Z and He X (2021) Ischemic-Free Liver Transplantation

Reduces the Recurrence of Hepatocellular Carcinoma After Liver Transplantation.

Front. Oncol. 11:773535. doi: 10.3389/fonc.2021.773535

\section{Ischemic-Free Liver Transplantation Reduces the Recurrence of Hepatocellular Carcinoma After Liver Transplantation}

\author{
Yunhua Tang ${ }^{1,2,3 \dagger}$, Tielong Wang ${ }^{1,2,3 \dagger}$, Weiqiang Ju ${ }^{1,2,3 \dagger}$, Fangcong $\mathrm{Li}^{1,2,3}$, Qi Zhang ${ }^{1,2,3}$, \\ Zhitao Chen 1,2,3, Jinlong Gong $^{1,2,3}$, Qiang Zhao ${ }^{1,2,3}$, Dongping Wang ${ }^{1,2,3}$, \\ Maogen Chen ${ }^{1,2,3^{*}}$, Zhiyong Guo ${ }^{1,2,3^{*}}$ and Xiaoshun $\mathrm{He}^{1,2,3^{*}}$ \\ 1 Organ Transplant Center, The First Affiliated Hospital, Sun Yat-sen University, Guangzhou, China, ${ }^{2}$ Guangdong Provincial \\ Key Laboratory of Organ Donation and Transplant Immunology, Guangzhou, China, ${ }^{3}$ Guangdong Provincial International \\ Cooperation Base of Science and Technology (Organ Transplantation), Guangzhou, China
}

Ischemia reperfusion injury (IRI) is an adverse factor for hepatocellular carcinoma (HCC) recurrence after liver transplantation. Ischemic-free liver transplantation (IFLT) is a novel transplant procedure that can largely reduce or even prevent IRI, but the clinical relevance of IFLT and the recurrence of HCC after liver transplantation are still unknown. This retrospective study compared survival outcomes, HCC recurrence, perioperative data and IRI severity following liver transplantation (LT). 30 patients received IFLT and 196 patients received conventional liver transplantation (CLT) were chosen for the entire cohort between June 2017 and August 2020. A 1:3 propensity score matching was performed, 30 IFLT recipients and 85 matched CLT patients were enrolled in propensity-matched cohorts. An univariate and multivariate Cox regression analysis was performed, and showed surgical procedure (CLT vs IFLT) was an independent prognostic factor ( $\mathrm{HR} 3.728,95 \% \mathrm{Cl} 1.172-11.861, P=0.026)$ for recurrence free survival (RFS) in HCC patients following liver transplantation. In the KaplanMeier analysis, the RFS rates at 1 and 3 years after $L T$ in recipients with $H C C$ in the IFLT group were significantly higher than those in the CLT group both in the entire cohort and propensitymatched cohort ( $P=0.006$ and $P=0.048$, respectively). In addition, patients in the IFLT group had a lower serum lactate level, lower serum ALT level and serum AST level on postoperative Day 1. LT recipients with HCC in the IFLT group had a lower incidence of early allograft dysfunction than LT recipients with HCC in the CLT group. Histological analysis showed no 
obvious hepatocyte necrosis or apoptosis in IFLT group. In conclusion, IFLT can significantly reduce $\mathrm{IRI}$ damage and has the potential to be a useful strategy to reduce HCC recurrence after liver transplantation.

Keywords: ischemia reperfusion injury, hepatocellular carcinoma, ischemic-free liver transplantation, prognosis, propensity-matched analysis

\section{INTRODUCTION}

Hepatocellular carcinoma (HCC) is the fifth most common cancer and the third cause of cancer-related mortality worldwide (1). Liver transplantation (LT) offers the most effective treatment for selected patients with HCC compared to liver resection or local ablation (2). However, the high incidence of postoperative recurrence has become a major concern and remains the main limitation of long-term outcomes of liver transplantation (3). Risk factors for HCC recurrence have been extensively investigated and are related to tumor size and number, microvascular invasion and poorly differentiated tumor grade $(4,5)$. In addition to tumor biology, increasing animal studies and clinical evidence suggest that ischemia reperfusion injury (IRI) promotes the recurrence of HCC after liver transplantation (6-8).

Liver grafts will inevitably be subject to varying degrees of IRI when organ procurement occurs after rapid cold flush, subsequent cold preservation and warm reperfusion after implantation into the recipient. Several studies have shown that liver IRI results in microvascular dysfunction, immune cell recruitment to liver grafts, and the release of pro-inflammatory and pro-proliferation mediators, facilitating the growth of circulating liver cancer cells in the injured liver $(9,10)$. For decades, great efforts have been made to reverse IRI and reduce the recurrence of HCC after liver transplantation, including ischemia preconditioning, immunological therapy and gene therapy $(6,11-13)$. However, few studies could be translated to the clinic. Obviously, none of the reported methods could effectively prevent IRI, which is an inevitable consequence due to cold preservation during liver transplantation. Ischemia-free liver transplantation (IFLT) is a novel transplant procedure that is able to procure, preserve and implant liver grafts without stopping the blood and oxygen supply for liver grafts. It has been well established that IRI was largely alleviated and even entirely prevented in IFLT in our previous studies $(14,15)$. However, to date, no reports have examined the clinical relevance of IFLT and the recurrence of HCC after liver transplantation.

Abbreviations: IRI, ischemia reperfusion injury; HCC, hepatocellular carcinoma; IFLT, ischemic-free liver transplantation; LT, liver transplantation; CLT, conventional liver transplantation; ALT, alanine aminotransferase; AST, aspartate aminotransferase; EAD, early allograft dysfunction; DBD, donation after brain death; DCD, donation after circulatory death; BMI, body mass index; MELD, Model for End-Stage Liver Disease; AFP, alpha-fetoprotein; RFA, radiofrequency ablation; TACE, transcatheter arterial chemoembolization; ICU, intensive care unit; SCS, static cold storage; HA, hepatic artery; NMP, normothermic machine perfusion; HE, hematoxylin and eosin; TUNEL, terminal deoxynucleotidyl transferase dUTP nick end labeling; SD, means \pm standard deviation; RFS, recurrence-free survival; OS, overall survival; PNF, primary nonfunctioning; POD, post-operative day.
In this study, we aimed to compare the transplant outcomes and graft IRI severity in recipients with HCC between IFLT and conventional liver transplantation (CLT), further assessing the impact of IFLT on the risk of HCC recurrence after liver transplantation.

\section{PATIENTS AND METHODS}

\section{Study Population}

This was a retrospective cohort design study. We included adult (18 years of age and older) patients diagnosed with HCC preoperatively who underwent CLT or IFLT at The First Affiliated Hospital of Sun Yat-sen University, Guangzhou, China, between June 2017 and August 2020. Split LT, liver-kidney combined transplantation or multivisceral transplantation were excluded, and patients who died within 30 days of LT were also excluded. Because patients who underwent IFLT only received donors from donation after brain death (DBD), donation after circulatory death (DCD) or living donors were excluded from the current study. Thus, patients identified as having HCC were chosen for the study population $(n=226) ; 30$ of 226 patients received IFLT, and the remaining 196 patients received CLT. All 226 donors were enrolled in a voluntary organ donation program for deceased Chinese citizens, and informed consent was obtained from relatives of the donors. No organ donations were from executed prisoners. This study was approved by the Institutional Review Board of the First Affiliated Hospital of Sun Yat-sen University.

The donor variables were age, BMI, donor serum creatinine, donor total bilirubin, donor serum sodium, and cold ischemia time. The recipient variables were age at transplant, BMI, preoperative laboratory MELD score, and positive hepatitis $\mathrm{B}$ surface antigen. Tumor parameters were pretransplant AFP, most radiologic tumor diameter, number of lesions $(1,2,3+)$, Milan criteria, liver resection history, neoadjuvant therapy (RFA or TACE), tumor differentiation (well, moderate, poor), and microvascular invasion. Intraoperative and posttransplantation data included operation duration, anhepatic phase, total blood loss, blood transfusion, ICU stay, hospital stay, early allograft dysfunction (EAD), serum INR, lactate, ALT, AST and creatinine level on the first day posttransplantation, recurrence, date of recurrence, and site of recurrence.

To evaluate whether the outcomes of HCC recipients who underwent IFLT or CLT were different, propensity-matched analyses were performed. IFLT recipients were matched 1:3 with patients who had undergone CLT during the same time period utilizing a propensity match score, matching for the following variables: pretransplant tumor characteristics (serum 
AFP, the most radiologic tumor diameter, multiple/single, portal vein tumor thrombosis, liver resection history, RFA/TACE neoadjuvant therapy) and explant tumor characteristics (differentiation and microvascular invasion). The caliper width was 0.2 standard deviations of the logit-transformed propensity score. The absolute standardized differences method was used to diagnose the balance after matching, and all were confirmed to be less than 0.25 .

The primary endpoint of this study was tumor recurrence. Secondary endpoints included operation time, intensive care and hospital stay, EAD, serum ALT and AST levels on the first day posttransplantation, histological analysis of liver tissues before procurement, at the end of preservation and after revascularization, and overall survival. EAD was defined by the presence of one or more of the following after LT (16): bilirubin of $\geq 10 \mathrm{mg} / \mathrm{dL}$ on Day 7 , international normalized ratio of $\geq 1.6$ on Day 7 , and alanine aminotransferase or aspartate aminotransferase $>2000$ IU/L within the first 7 days. To estimate overall survival, survival time was calculated from the date of LT to death or last known follow-up, and status was recorded at the last point of contact with the patient who died or lived. To estimate recurrence-free survival (RFS), patients with no evidence of recurrence were censored at the time, of last follow-up or death.

\section{CLT and IFLT Procedure}

The CLT procedure included organ procurement after rapid cold flush, subsequent static cold storage (SCS) and back-table preparation, and then implantation. The surgical procedures of IFLT were as described in our previous study $(14,15)$. Briefly, after the liver was fully mobilized, a $4 \mathrm{~cm}$-long segment of the external iliac vein from the blood donor was harvested and endto-side anastomosed to the portal vein of liver donors, serving as an access point for portal vein cannulation while still permitting native blood flow through the portal vein. A 12 Fr cannula was inserted into the splenic artery (or gastroduodenal artery) without interruption of arterial supply to the liver from the celiac artery. A 32 Fr cannula was placed in the infrahepatic inferior vena cava for outflow. A straight 24 Fr cannula connected to the portal vein perfusion line of Liver Assist (Organ Assist, Groningen, The Netherlands) was inserted into the portal vein via the interposition vein. The arterial cannula was then connected to the hepatic artery (HA) perfusion line of
Liver Assist. After the in situ normothermic mechanical perfusion (NMP) circuit for the livers was established, the livers were harvested and moved to the organ reservoir under continuous NMP. So that the graft did not suffer ischemia during procurement. Hereafter, the liver underwent ex situ NMP. The perfusate contained approximately $1.3 \mathrm{~L}$ cross-matched leucocyte-depleted washed red cells, $1.4 \mathrm{~L}$ Succinylated gelatinor, $30 \mathrm{~mL} 5 \%$ sodium bicarbonate, $0.5 \mathrm{~g}$ metronidazole, $37500 \mathrm{U}$ heparin, $1.5 \mathrm{~g}$ cefoperazone sodium and sulbactam sodium, $30 \mathrm{~mL} \mathrm{10 \%} \mathrm{calcium} \mathrm{gluconate,} 3 \mathrm{~mL} 25 \%$ magnesium sulfate and $250 \mathrm{~mL}$ compound amino acid injection. In IFLT, no back-table preparation is needed, and the liver is implanted under continuous NMP. Briefly, the splenic artery (or gastroduodenal artery) and interposition vein and all vascular anastomoses (donor suprahepatic vena cava to recipient suprahepatic vena cava, donor portal vein to recipient portal vein, and donor celiac artery/common hepatic artery to recipient common hepatic artery) were performed without interruption of the blood supply to the graft under continuous NMP. After the native blood supply from the recipient's portal vein and hepatic artery to the graft was re-established, the in situ NMP was stopped, and the cannulas were removed. The donor splenic artery (or gastroduodenal artery) was ligated closed, and the interposition vein was sutured closed. The donor infrahepatic vena cava and common bile duct were anastomosed to the recipient counterparts. Graphic rendering of the ischemia-free liver transplantation procedure is shown in Figure 1.

\section{Sample Collection}

In ex vivo perfusion, samples from the perfusate were collected for analysis of the blood gas parameters (pO2, pCO2, $\mathrm{PH}$ and lactate). Perfusate samples were also collected and centrifuged, and the supernatant was stored at $-80^{\circ} \mathrm{C}$ for liver function tests, such as aspartate aminotransferase (AST), alanine aminotransferase (ALT), and total bilirubin (Tbil), using standard biochemical methods. Bile production was collected every 60 minutes from the biliary draining tube for bile production calculation and detection of $\mathrm{PH}$ and $\mathrm{HCO}^{-}$of bile. Liver tissue biopsy was performed for histology studies before procurement, at the end of preservation and after revascularization, including hematoxylin and eosin (HE) staining for histological scoring and terminal deoxynucleotidyl transferase dUTP nick end labeling (TUNEL) assay for hepatocyte apoptosis.

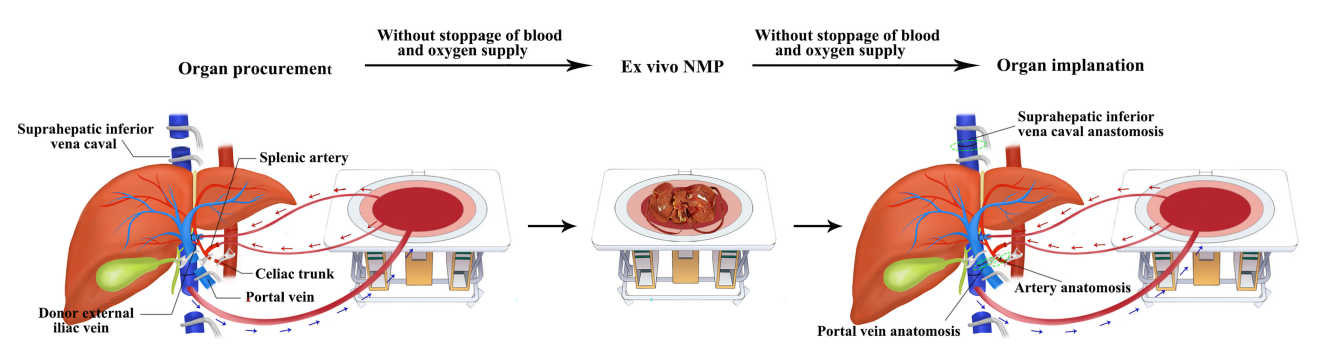

FIGURE 1 | Graphic rendering of the ischemia-free liver transplantation procedure. 


\section{Transplantation Procedures and Postoperative Management}

After CLT or IFLT, all patients were admitted to the intensive care unit. The immunosuppressive regimen was $20 \mathrm{mg}$ basiliximab induction therapy administered during the operation and on postoperative Day 4. Both a calcineurin inhibitor and mycophenolate mofetil were administered for immunosuppressive maintenance therapy beginning on postoperative Day 4 .

\section{Statistical Analysis}

The donor and recipient characteristics were expressed as the means \pm standard deviation (SD) for metric parameters and as percentages for nominal parameters. Continuous data were compared using ttests, whereas categorical variables were compared using the chisquare test or Fisher's exact test. We used Kaplan-Meier statistics and the log-rank test to analyze the recurrence-free survival (RFS) and overall survival (OS) rates of the patients both in the entire cohort and propensity-matched cohorts. To identify independent predictors of RFS in entire cohort, an univariate and multivariate Cox proportional hazard regression was conducted for all candidate predictors of RFS. Pretransplant serum AFP levels were categorized into two groups according to interquartile range 3 (IQR3). Variables with a $P<0.05$ in univariate analysis were subjected to multivariate Cox proportional hazards regression via the forward stepwise method; the results were presented as hazard ratios (HRs) with
95\% confidence intervals (CIs). The data analysis was performed using STATA 14.0 software (Stata Corp). $P<0.05$ was considered to be statistically significant.

\section{RESULTS}

\section{Recipient and Donor Clinical Characteristics Between the IFLT group and CLT Group}

Patients identified as having HCC were chosen for the study population $(n=226)$ between June 2017 and August 2020 in the entire cohort; 30 of 226 patients received IFLT, and the remaining 196 patients received CLT. To correct selection biases and confounding factors, propensity score matching was performed at a 1:3 ratio. After matching, 30 IFLT recipients and 85 matched CLT patients from our center were enrolled in propensity-matched cohorts during the same period. In the entire cohort, the duration of follow-up was $22.9 \pm 11.2$ months in the IFLT group and $22.6 \pm 11.4$ months in the CLT group with no significant differences, securing a minimal followup of 6 months in the two groups.

There were no significant differences in donor and recipient characteristics between the IFLT group and the CLT group both in the entire cohort and in the propensity-matched cohort, including

TABLE 1 | Recipient and donor characteristics in IFLT and CLT groups before and after propensity score matching.

\begin{tabular}{|c|c|c|c|c|c|c|}
\hline & \multicolumn{3}{|c|}{ Entire cohort } & \multicolumn{3}{|c|}{ Propensity-matched cohort } \\
\hline & IFLT group $(n=30)$ & CLT group $(n=196)$ & $\mathbf{P}$ & IFLT group $(n=30)$ & CLT group $(n=85)$ & $\mathbf{P}$ \\
\hline \multicolumn{7}{|l|}{ Donor Characteristics } \\
\hline Donor age (years) & $41.4 \pm 14.2$ & $36.8 \pm 14.9$ & 0.117 & $41.4 \pm 14.2$ & $35.1 \pm 14.3$ & 0.053 \\
\hline Gender: male & $66.7 \%(20 / 30)$ & $74.0 \%(145 / 196)$ & 0.401 & $66.7 \%(20 / 30$ & $71.8 \%(61 / 85)$ & 0.599 \\
\hline BMl, $\mathrm{kg} / \mathrm{m}^{2}$ & $22.3 \pm 2.3$ & $23.3 \pm 9.8$ & 0.771 & $22.3 \pm 2.3$ & $22.2 \pm 3.1$ & 0.896 \\
\hline Donor serum creatinine (ummol/L) & $87.3 \pm 67.2$ & $139.2 \pm 155.6$ & 0.074 & $87.3 \pm 67.2$ & $125.6 \pm 107.7$ & 0.071 \\
\hline Donor total bilirubin (umol/L) & $27.7 \pm 21.5$ & $23.4 \pm 17.6$ & 0.250 & $27.7 \pm 21.5$ & $22.9 \pm 14.5$ & 0.180 \\
\hline Donor serum sodium (mmol/L) & $147.6 \pm 12.6$ & $149.7 \pm 16.7$ & 0.508 & $147.6 \pm 12.6$ & $148.3 \pm 20.8$ & 0.870 \\
\hline Cold ischemia time (hours) & NA & $6.7 \pm 2.1$ & NA & NA & $6.8 \pm 2.2$ & NA \\
\hline \multicolumn{7}{|l|}{ Recipient Characteristics } \\
\hline Age at transplant (years) & $54.2 \pm 9.9$ & $50.2 \pm 73$ & 0.088 & $54.2 \pm 9.9$ & $50.74 \pm 9.9$ & 0.106 \\
\hline Gender: male & $96.7 \%(29 / 30)$ & $92.9 \%(182 / 196)$ & 0.435 & $96.7 \%(29 / 30)$ & $90.6 \%(77 / 85)$ & 0.287 \\
\hline $\mathrm{BMl}, \mathrm{kg} / \mathrm{m}^{2}$ & $23.4 \pm 3.1$ & $23.2 \pm 3.2$ & 0.793 & $23.4 \pm 3.1$ & $23.5 \pm 3.5$ & 0.822 \\
\hline Preoperative lab MELD score & $15.4 \pm 7.7$ & $13.7 \pm 8.1$ & 0.279 & $15.4 \pm 7.7$ & $13 \pm 7.8$ & 0.174 \\
\hline Positive Hepatitis B surface antigen & $90.0 \%(27 / 30)$ & $86.7 \%(170 / 196)$ & 0.618 & $90.0 \%(27 / 30)$ & $85.9 \%(73 / 85)$ & 0.565 \\
\hline \multicolumn{7}{|l|}{ Tumor parameter } \\
\hline Pretransplant AFP (ug/l) & $167.9 \pm 428.5$ & $12899.4 \pm 729.2$ & 0.016 & $167.9 \pm 428.5$ & $97.9 \pm 342.8$ & 0.371 \\
\hline Size biggest HCC lesion (mm) & $43.47 \pm 16.7$ & $54.23 \pm 41.74$ & 0.129 & $43.47 \pm 16.7$ & $44.5 \pm 33.8$ & 0.751 \\
\hline \multicolumn{7}{|l|}{ Number of lesions } \\
\hline single & $46.7 \%(14 / 30)$ & $39.3 \%(77 / 196)$ & 0.443 & $46.7 \%(14 / 30)$ & $42.4 \%(36 / 85)$ & 0.682 \\
\hline Multiple & $53.3 \%(16 / 30)$ & $60.7 \%(119 / 196)$ & & $53.3 \%(16 / 30)$ & $57.6 \%(49 / 85)$ & \\
\hline $\begin{array}{l}\text { Within Milan criteria } \\
\text { tumor differentiation }\end{array}$ & $56.7 \%(17 / 30)$ & $36.2 \%(71 / 196)$ & 0.032 & $56.7 \%(17 / 30)$ & $48.2 \%(41 / 85)$ & 0.427 \\
\hline Well & $3.3 \%(1 / 30)$ & $3.1 \%(6 / 196)$ & 0.994 & $3.3 \%(1 / 30)$ & $4.7 \%(4 / 85)$ & 0.839 \\
\hline Moderate & $73.3 \%(22 / 30)$ & $73.0 \% \%(143 / 196)$ & & $73.3 \%(22 / 30)$ & $76.5 \%(65 / 85)$ & \\
\hline Poor & $23.3 \%(7 / 30)$ & $24.0 \%(47 / 196)$ & & $23.3 \%(7 / 30)$ & $18.8 \%(16 / 85)$ & \\
\hline Microvascular invasion & $16.7 \%(5 / 30)$ & $33.7 \%(66 / 196)$ & 0.042 & $16.7 \%(5 / 30)$ & $18.8 \%(16 / 85)$ & 0.793 \\
\hline Liver resection history & $23.3 \%(7 / 30)$ & $17.9 \%(35 / 196)$ & 0.772 & $23.3 \%(7 / 30)$ & $21.2 \%(18 / 85)$ & 0.806 \\
\hline neoadjuvant therapy (RFA or TACE) & $46.7 \%(14 / 30)$ & $52.0 \%(102 / 196)$ & 0.328 & $46.7 \%(14 / 30)$ & $52.9 \%(45 / 85)$ & 0.554 \\
\hline Duration of follow-up (days) & $22.9 \pm 11.2$ & $22.6 \pm 11.4$ & 0.917 & $22.9 \pm 11.2$ & $24.8 \pm 11.9$ & 0.441 \\
\hline
\end{tabular}

The bold emphasis of $P$ value means that the value less than 0.05 has statistical significance. 
donor characteristics (age, gender, donor BMI, donor serum creatinine, donor serum total bilirubin and donor serum sodium) and recipient characteristics (age, gender, BMI, laboratory MELD scores and positive hepatitis B surface antigen). The cold ischemia time in the CLT group was $6.7 \pm$ 2.1 hours in the entire cohort and $6.8 \pm 2.2$ hours in the propensity-matched cohort. Donor and recipient characteristics were summarized in Table $\mathbf{1 .}$

\section{Comparison of Tumor Parameters Between the IFLT Group and CLT Group}

In the entire cohort, the pretransplant AFP level was higher in the CLT group than in the IFLT group $(P=0.016)$. The percentage of LT recipients within the Milan criteria and microvascular invasion was higher in the CLT group than in the IFLT group ( $P=0.032$ and $P=0.042$, respectively). There were no differences in the size largest HCC lesion, number of lesions, tumor differentiation, liver resection history and neoadjuvant therapy (RFA or TACE) history between the two groups. In the propensity-matched cohort, there were no differences in all tumor parameters between the two groups. Comparison of tumor parameters were summarized in Table $\mathbf{1 .}$

\section{IFLT Provides a Larger Benefit for the Reduction in Post-LT HCC Recurrence Than CLT}

To analysis whether IFLT has potential as an independent prognostic factor in HCC patients following liver transplantation, Cox regression analysis was performed to examine RFS in the entire cohort. Univariate analysis indicated that pretransplant AFP $(\geq 300$ ug/l vs. $<300 \mathrm{ug} / \mathrm{l})(\mathrm{HR} 3.830,95 \%$ CI 2.447-5.997, $P<0.001)$, biggest HCC diameter $(\geq 5 \mathrm{~cm} v s .<5 \mathrm{~cm})$ (HR 1.753, 95\% CI 1.119-2.746, $P$ $=0.014$ ), poor tumor differentiation (HR 2.738, 95\% CI 1.226-6.112, $P=0.014$ ), microvascular invasion (HR 3.453, 95\% CI 2.213-5.388, $P<0.001)$ and surgical procedure (CLT vs IFLT) (HR 4.371, 95\% CI 1.371-13.864, $P=0.012$ ) were associated with RFS. Furthermore, multivariate analysis demonstrated that pretransplant AFP $(\geq 300$ ug/l vs. $<300 \mathrm{ug} / \mathrm{l})(\mathrm{HR} 2.262,95 \%$ CI 1.597-4.318, $P<0.001)$, microvascular invasion (HR 2.309, 95\% CI 1.403-3.801, $P<0.001$ ) and surgical procedure (CLT vs IFLT) (HR 3.728, 95\% CI 1.17211.861, $P=0.026$ ) were independent prognostic factors for DFS in HCC patients following liver transplantation (Table 2).

In the entire cohort, Kaplan-Meier analysis showed the RFS rates at 1 and 3 years after LT in recipients with HCC in the IFLT group were $92.2 \%$ and $86.7 \%$, respectively, which were significantly higher than those $(73.0 \%$ and $46.3 \%)$ in the CLT group $(P=0.006$, Figure 2A). The overall survival rates at 1 and 3 years after LT in recipients with HCC in the IFLT group were $96.7 \%$ and $90.6 \%$, respectively, which tended to be higher than those $(90.2 \%$ and $68.1 \%)$ in the CLT group, but with no significant differences $(P=0.089$, Figure 2B). In the propensitymatched cohort, the RFS rates at 1 and 3 years after LT in recipients with HCC in the IFLT group were $92.2 \%$ and $86.7 \%$, respectively, which were significantly higher than those $(88.1 \%$ and $53.6 \%$, respectively) in the CLT group ( $P=0.048$, Figure 2C). The overall survival rates at 1 and 3 years after LT in recipients with HCC in the IFLT group were $96.7 \%$ and $90.6 \%$, respectively, which tended to be higher than those $(94.1 \%$ and $70.6 \%)$ in the CLT group, but with no significant differences $(P=0.442$, Figure 2D). These results indicate that IFLT provides greater benefits than CLT in terms of the reduction in post-LT HCC recurrence.

TABLE 2 | Univariate and multivariate analyses of risk factors for recurrence-free survival in the entire cohort.

\begin{tabular}{|c|c|c|c|c|c|c|}
\hline & \multicolumn{3}{|c|}{ Univariate analysis } & \multicolumn{3}{|c|}{ Multivariate analysis } \\
\hline & HR & $95 \% \mathrm{Cl}$ & $\mathbf{P}$ & HR & $95 \% \mathrm{Cl}$ & $\mathbf{P}$ \\
\hline \multicolumn{7}{|l|}{ Recipient Characteristics } \\
\hline age at transplant (years) & 0.980 & $0.959-1.001$ & 0.062 & & & \\
\hline Gender: male vs female & 1.030 & $0.448-2.371$ & 0.944 & & & \\
\hline $\mathrm{BMI}, \mathrm{kg} / \mathrm{m}^{2}$ & 1.006 & $0.940-1.077$ & 0.860 & & & \\
\hline Preoperative lab MELD score & 1.008 & $0.979-1.037$ & 0.599 & & & \\
\hline Positive Hepatitis B surface antigen & 1.382 & $0.418-4.569$ & 0.596 & & & \\
\hline Pretransplant AFP: $\geq 300$ vs. $<300$ ug/l & 3.830 & $2.447-5.997$ & $<0.001$ & 2.626 & $1.597-4.318$ & $<0.001$ \\
\hline biggest HCC diameter: $\geq 5 \mathrm{~cm}$ vs. $<5 \mathrm{~cm}$ & 1.753 & $1.119-2.746$ & 0.014 & & & \\
\hline Tumor Number ( single vs. multiple) & 1.009 & $0.643-1.582$ & 0.969 & & & \\
\hline Tumor differentiation: Moderate vs. Well & 1.702 & $0.801-3.614$ & 0.167 & & & \\
\hline Tumor differentiation: Poor vs. Well & 2.738 & $1.226-6.112$ & 0.014 & & & \\
\hline Microvascular invasion & 3.453 & $2.213-5.388$ & $<0.001$ & 2.309 & $1.403-3.801$ & 0.001 \\
\hline Liver resection history & 0.983 & $0.560-1.725$ & 0.952 & & & \\
\hline Neoadjuvant therapy (RFA or TACE) & 1.113 & $0.715-1.732$ & 0.636 & & & \\
\hline \multicolumn{7}{|l|}{ Donor Characteristics } \\
\hline Donor age (years) & 1.002 & $0.987-1.017$ & 0.824 & & & \\
\hline $\mathrm{BMl}, \mathrm{kg} / \mathrm{m}^{2}$ & 0.946 & $0.878-1.019$ & 0.144 & & & \\
\hline Donor serum creatinine (ummol/L) & 1.001 & $0.999-1.003$ & 0.393 & & & \\
\hline Donor total bilirubin (umol/L) & 0.994 & $0.980-1.008$ & 0.382 & & & \\
\hline Donor serum sodium (mmol/L) & 1.001 & $0.988-1.014$ & 0.899 & & & \\
\hline surgical procedure: CLT vs. IFLT & 4.371 & $1.371-13.864$ & 0.012 & 3.728 & $1.172-11.861$ & 0.026 \\
\hline
\end{tabular}

The bold emphasis of $P$ value means that the value less than 0.05 has statistical significance. 

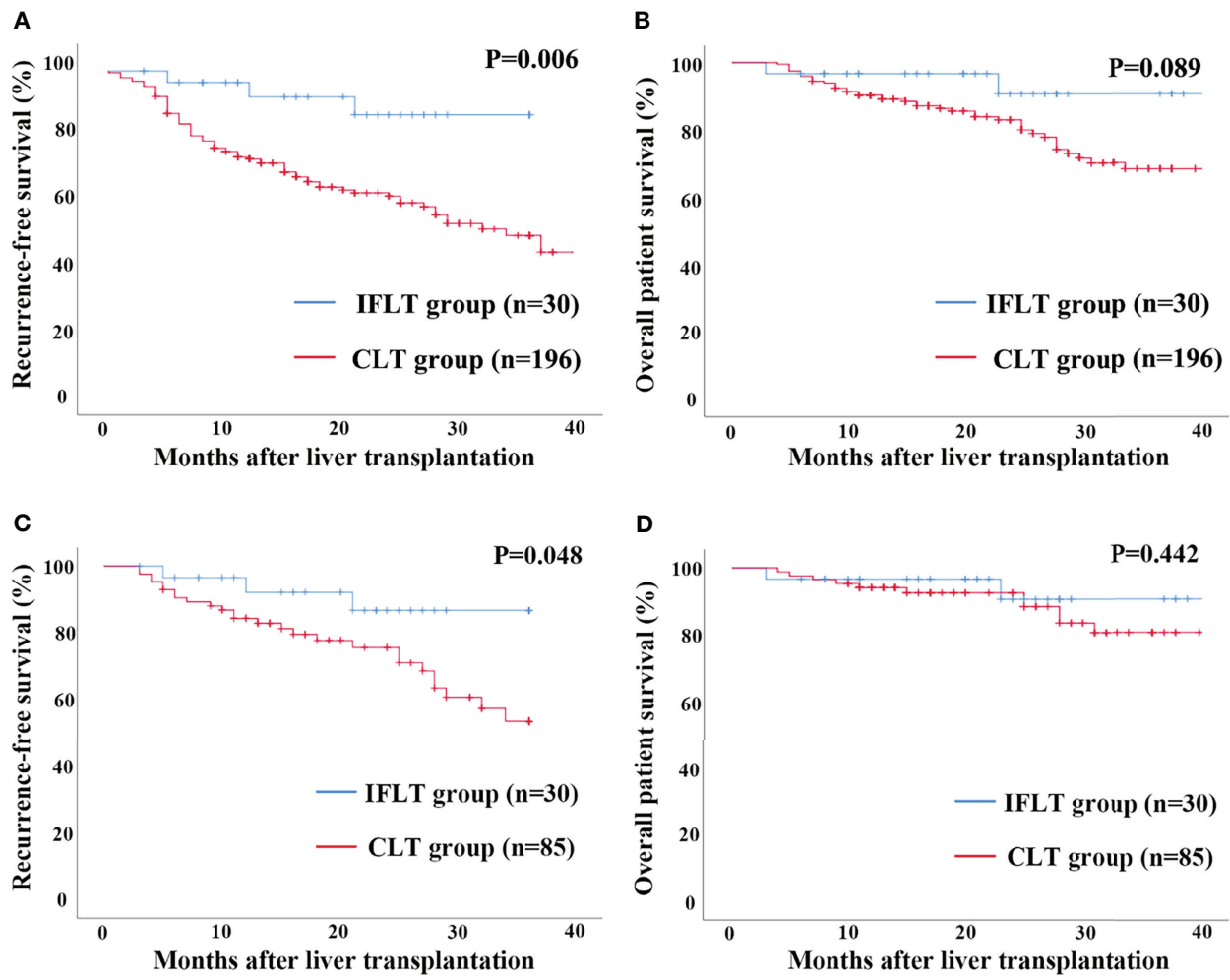

FIGURE 2 | Recurrence-free survival and overall survival by Kaplan-Meier survival analysis for patients transplanted for HCC between the IFLT and CLT group for 3 years' follow-up. (A) Recurrence-free survival in the entire cohort. (B) Overall survival in the entire cohort. (C) Recurrence-free survival in the propensity-matched cohort. (D) Overall survival in the propensity-matched cohort.

\section{Comparison of Operative and Postoperative Outcomes Between the IFLT Group and CLT Group}

In the entire cohort, LT recipients with HCC in the IFLT group had a shorter operation duration than LT recipients with HCC in the CLT group ( $6.3 \pm 1.4$ hours vs. $6.9 \pm 1.5$ hours, $P=0.016$ ). The anhepatic time was not different between the IFLT and CLT groups $(52.2 \pm 16.9$ mins vs. $53.3 \pm 14.8$ mins, $P=0.980)$. There were no differences in total blood loss or blood transfusion between the two groups. The ICU stay $(52.4 \pm 50.7$ hours $v$ s. $53.8 \pm 50.2$ hours, $P=0.895)$ and hospital stay $(23.8 \pm 17.6$ days $v s$. $25.8 \pm 15.1$ days, $P=0.772$ ) were similar between the two groups. LT recipients with HCC in the IFLT group had a lower incidence of EAD than LT recipients with HCC in the CLT group (3.3\% vs. $29.6 \%, P=0.002)$. Patients in the IFLT group had a lower serum lactate level $(1.9 \pm 1.2 \mathrm{mmol} / \mathrm{L} v s .2 .7 \pm 1.3 \mathrm{mmol} / \mathrm{L}, P=0.005)$, lower serum ALT level $(198.8 \pm 157.9 \mathrm{U} / \mathrm{L}$ vs. $633.8 \pm 706.2 \mathrm{U} / \mathrm{L}$, $P=0.001)$ and serum AST level $(437.1 \pm 328.9$ U/L vs. $1571.6 \pm$ 1764.6 U/L, $P=0.001$ ) on postoperative Day 1. There were no differences in serum creatine levels or serum INR levels between the two groups on postoperative Day 1.

In the propensity-matched cohort, the indicators mentioned above had the same trend. Operative and postoperative outcomes are summarized in Table 3.

\section{IFLT Is Feasible, Safe and Effective, and Can Largely Reduce IRI in LT Recipients With HCC}

The NMP device provided adequate $\mathrm{O} 2$ and extraction of $\mathrm{CO} 2$ of the perfusion fluid with stable pressure and flow of both the portal vein and hepatic artery throughout the whole IFLT procedure (Figures 3A, B). The lactate levels in the perfusate dropped quickly from $4.73 \pm 2.22 \mathrm{mmol} / \mathrm{L}$ to normal, and the $\mathrm{pH}$ value in the perfusate was within the normal physiological range, reflecting active metabolism by the liver grafts (Figure 3C). The liver grafts presented a vivid appearance during procurement, ex vivo NMP and implantation (Figure 3D). Continuous bile production with a high sodium bicarbonate and $\mathrm{pH}$ level indicated good quality of the bile (Figures 3E, F). Altogether, these results indicate the effectiveness of IFLT and suggest excellent organ viability.

Hematoxylin and eosin (HE) staining evaluation of IFLT allograft biopsies showed stable and low Suzuki scores, whereas increased Suzuki scores were observed in the CLT allograft biopsies at the end of preservation graft and after revascularization (Figure 4A). The TUNEL assay showed no significant increase in apoptotic hepatocytes throughout the whole IFLT procedure in the IFLT group, while a significant increase in apoptotic hepatocytes was observed at the end of the 
TABLE 3 | Operative and postoperative outcomes in IFLT and CLT groups before and after propensity score matching.

\begin{tabular}{|c|c|c|c|c|c|c|}
\hline & \multicolumn{3}{|c|}{ Entire cohort } & \multicolumn{3}{|c|}{ Propensity-matched cohort } \\
\hline & IFLT group $(n=30)$ & CLT group $(n=196)$ & $\mathbf{P}$ & IFLT group $(n=30)$ & CLT group $(n=85)$ & $\mathbf{P}$ \\
\hline Operation duration (hours) & $6.3 \pm 1.4$ & $6.9 \pm 1.5$ & 0.016 & $6.3 \pm 1.4$ & $7.0 \pm 1.4$ & 0.017 \\
\hline Anhepatic phase (mins) & $52.2 \pm 16.9$ & $53.3 \pm 14.8$ & 0.980 & $52.2 \pm 16.9$ & $52.7 \pm 13.2$ & 0.086 \\
\hline Total blood loss (mL) & $1726 \pm 830$ & $1626 \pm 1649$ & 0.775 & $1726 \pm 830$ & $1418 \pm 1069$ & 0.259 \\
\hline Blood transfusion (ml) & $960 \pm 860$ & $940 \pm 960$ & 0.908 & $960 \pm 860$ & $820 \pm 780$ & 0.395 \\
\hline ICU stay (h) & $52.4 \pm 50.7$ & $53.8 \pm 50.2$ & 0.895 & $52.4 \pm 50.7$ & $55.1 \pm 47.2$ & 0.798 \\
\hline Hospital stay (day) & $23.8 \pm 17.6$ & $25.8 \pm 15.1$ & 0.772 & $23.8 \pm 17.6$ & $24.6 \pm 14.1$ & 0.816 \\
\hline EAD & $3.3 \%(1 / 30)$ & $29.6 \%(58 / 196)$ & 0.002 & $3.3 \%(1 / 30)$ & $29.4 \%(25 / 85)$ & 0.003 \\
\hline INR day 1 & $1.5 \pm 0.4$ & $1.4 \pm 0.3$ & 0.179 & $1.5 \pm 0.4$ & $1.5 \pm 0.3$ & 0.575 \\
\hline Serum lactate day 1 & $1.9 \pm 1.2$ & $2.7 \pm 1.3$ & 0.005 & $1.9 \pm 1.2$ & $2.6 \pm 1.3$ & 0.021 \\
\hline ALT day $1, \mathrm{U} / \mathrm{L}$ & $198.8 \pm 157.9$ & $633.8 \pm 706.2$ & 0.001 & $198.8 \pm 157.9$ & $617.6 \pm 819.5$ & 0.007 \\
\hline AST day $1, \mathrm{U} / \mathrm{L}$ & $437.1 \pm 328.9$ & $1571.6 \pm 1764.6$ & 0.001 & $437.1 \pm 328.9$ & $1393 \pm 1610$ & 0.002 \\
\hline Creatinine day $1, \mathrm{mmol} / \mathrm{L}$ & $95.7 \pm 43.2$ & $84.8 \pm 35.3$ & 0.129 & $95.7 \pm 43.2$ & $84.3 \pm 37.3$ & 0.186 \\
\hline
\end{tabular}

The bold emphasis of $P$ value means that the value less than 0.05 has statistical significance.

preservation graft and after revascularization (Figure 4B). These results suggest that IFLT can largely reduce IRI in LT recipients with HCC.

\section{DISCUSSION}

IRI is an unavoidable adverse factor in liver transplantation. IRI can impair the function of transplanted organs, leading to EAD or even primary nonfunctioning (PNF), increasing the incidence of complications and mortality in recipients after surgery (17). In addition, IRI is associated with tumor recurrence and metastasis after liver transplantation. Much effort has been devoted to reducing the degree of IRI, including ischemia preconditioning and the use of protective gases, stem cells or gene therapy. However, few methods have been translated into clinical practice (18). Recently, great achievements have been made in ex vivo machine perfusion of organs. In particular, NMP could offer oxygen and nutrients and allow functional testing of liver grafts, which have been demonstrated to be obviously superior to static cold storage in minimizing IRI (19). In the current NMP setting, the organs still suffer cold storage injury and ischemia due to cold flush before procurement, back-table preparation on ice and normal saline flush after NMP. Research has shown that a short period of cold ischemia time still results in significant sinusoidal endothelial cell dysfunction and Kupffer cell activation in the liver (20). Therefore, we established IFLT without stopping the blood and oxygen supply and any cold preservation for the

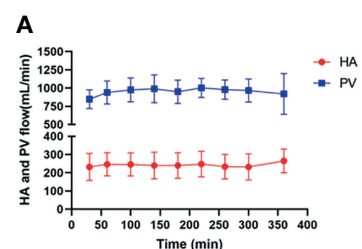

\section{B}

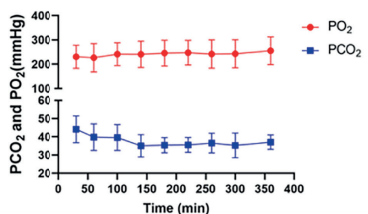

D

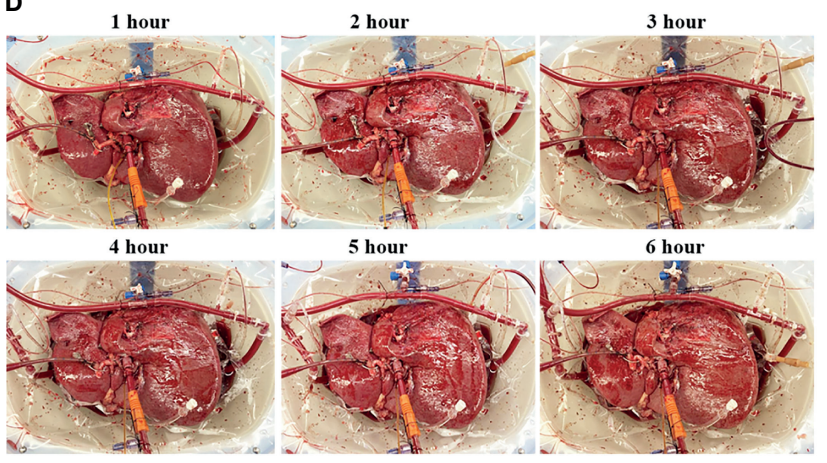

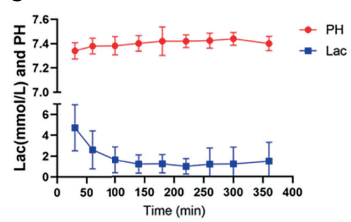

E
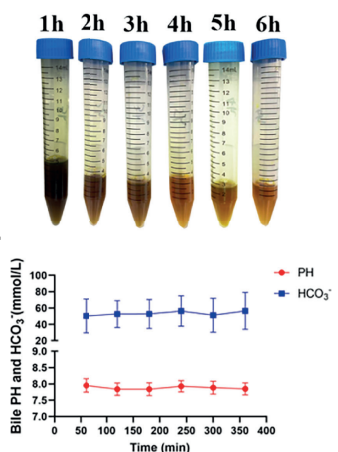

FIGURE 3 | Normothermic machine perfusion. (A) The arterial and portal venous flow rates; (B) The O2 and CO2 tension in the perfusate. (C) pH values and lactate levels in the perfusate. (D) The grafts presented a vivid appearance during ex vivo perfusion; (E) The produced bile during ex vivo perfusion; (F) PH value and bicarbonate levels of the produced bile. 

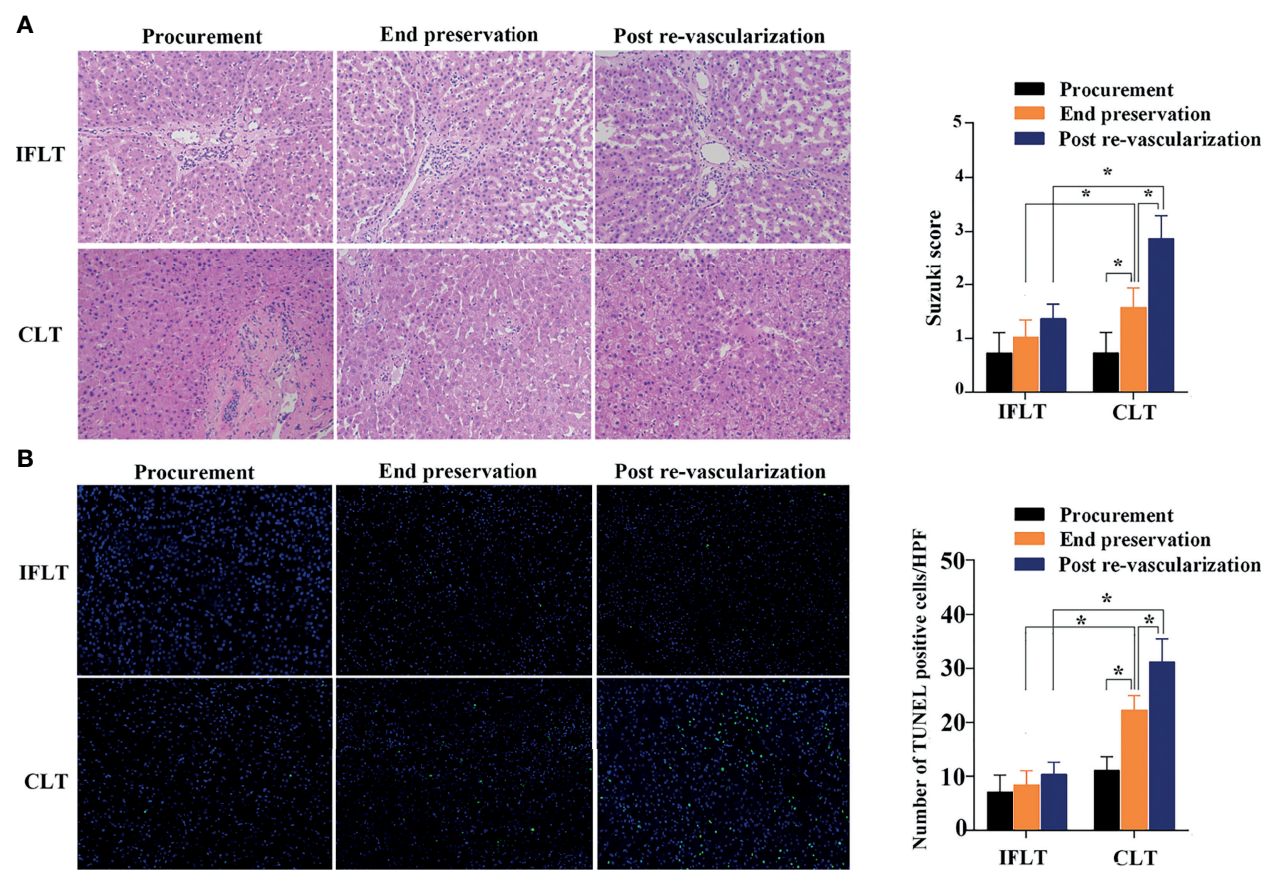

FIGURE 4 | Histological analysis of liver tissues. (A) Hematoxylin and eosin (HE) of donor liver tissue biopsies before procurement, at the end of preservation and post-reperfusion in the IFLT and CLT. (B) The TdT-mediated dUTP nick end labelling (TUNEL) assay revealed that the number of apoptotic hepatocytes per high power field (HPF) before procurement, at the end of preservation and post-reperfusion in the IFLT and CLT. ${ }^{\star} P<0.001$.

liver grafts during the whole transplant procedure. The protective effect of IFLT in reducing IRI on the donor liver was obvious. Peak transaminase levels within 1 week after LT correlated with both cold ischemia time and warm ischemia time, which can reflect the severity of IRI (21) and is regarded as a well-defined surrogate marker for long-term graft function and survival (21). In the current study, the peak value of transaminase on posttransplant Day 1 in the IFLT group was significantly lower than that in the CLT group. In addition, EAD suggests the initial poor function of liver grafts and represents the clinical phenotype of severe IRI after liver transplantation. Our results showed that LT recipients with HCC in the IFLT group had a lower incidence of EAD than LT recipients with HCC in the CLT group (3.3\% vs. $29.4 \%, P=0.003$ ), which was also much lower than the incidence of EAD of $20.0-30.0 \%$ reported by other centers in Western countries (16, 22, 23). Furthermore, histological analysis showed no significant increase in the Suzuki score or apoptotic hepatocytes during the whole transplant procedure in the IFLT group. In the IFLT group, the amount of blood loss and blood transfusion did not increase, and the postoperative ICU hospital stay and total hospital stay did not increase. These results suggested that IFLT did not increase the difficulty and complexity of the operation, nor did it increase the complications and hospitalization costs. Together, these findings indicate that IRI can be largely prevented in IFLT.

Clinically, previous studies have shown that posttransplant cancer recurrence and metastasis are significantly correlated with many factors, including tumor size and number (24), microvascular invasion (25), elevated AFP level $(26,27)$ and poorly differentiated tumor grade (4). In addition to liver tumor biology itself, increasing evidence supports the adverse effect of liver ischemia on the risk of liver cancer recurrence after liver transplantation. Nagai et al. (28) showed that prolonged cold and warm ischemia times of the liver graft are independent predictors of HCC recurrence one year after liver transplantation. Orci et al. (29) confirmed that LT recipients of organs from DCD donors with long warm ischemia times had higher HCC recurrence after liver transplantation. Several transplantation centers have reported that the rate of HCC recurrence was significantly higher following living donor liver transplantation than following deceased donor liver transplantation because the liver graft from a living donor is usually small for the recipient and more vulnerable to IRI $(30,31)$. On the other hand, several attempts targeting graft IRI effectively decreased the risk of early HCC recurrence after liver transplantation. Kornberg et al. (6) reported that treating liver graft IRI with prostaglandin E1 significantly increased the recurrence-free survival rates of recipients with HCC. A mouse model mimicking the recurrence of HCC after liver transplantation suggested that remote ischemic preconditioning offers protection against ischemia-mediated accelerated HCC recurrence (8). Matteo et al. (32) reported that the performance of hypothermic oxygenated liver perfusion before liver implantation appears advantageous to protect against HCC recurrence after liver transplantation, despite extended tumor criteria. In the current study, IRI was largely prevented. Here, we compared the 
difference in the postoperative recurrence rate between the IFLT and CLT groups. To correct selection biases and confounding factors, propensity score matching was performed. The tumor parameters, including tumor size, number, AFP level, tumor grade, microvascular infiltration and preoperative downstage treatment, were consistent between the two groups in our study. Both groups of patients received DBD liver donation without warm ischemia time, and IRI can be largely reduced even completely prevented by performing IFLT. An univariate and multivariate Cox regression analysis was performed in our study, and showed surgical procedure (CLT vs IFLT) was an independent prognostic factor for RFS in HCC patients following liver transplantation. In the Kaplan-Meier analysis, the recurrence free survival rates at 1 and 3 years after LT in recipients with HCC in the IFLT group were significantly higher than those in the CLT group both in the entire cohort and propensity-matched cohort $(\mathrm{P}=0.006$ and $\mathrm{P}=0.048$, respectively). These results together indicated that IFLT provides greater benefit than CLT in terms of the reduction in post-LT HCC recurrence, which supports that IRI had an impact on tumor recurrence after liver transplantation and that preventing or reducing IRI can reduce the HCC recurrence rate after liver transplantation.

The significance of IFLT in liver transplantation for HCC may not only improve the prognosis but also may expand the donor pool. Organ shortages are a problem to be solved worldwide, and patients with HCC who exceed the Milan or UCSF standard rarely have the opportunity to obtain a suitable liver. Marginal organs have greater vulnerability to IRI, such as older donors and fatty livers (33). Because China still in its early stages in developing an organ donation system based on deceased citizens, most donations originate from primary care hospitals, because of the lack of both basic medical equipment and doctors experienced with managing donors, these donors often suffer from multiple risk factors such as unstable blood circulation, hypoproteinemia, infection, and electrolyte disturbance. To be honest, we are relatively cautious about donor selection. Therefore, our initial experience is often based with a very young donors, which is a limitation of the study. However, IFLT can protect these marginal donor livers from IRI damage. Our previous studies reported the successful use of marginal donor livers (such as with hyperbilirubinemia or $85-95 \%$ macrovesicular steatosis) in LT recipients using IFLT $(14,34)$. Therefore, we recommend increasing the utilization of extended donor criteria or marginal donor liver grafts in recipients with HCC by performing IFLT in the future due to the benefit of IFLT-mediated reduction in IRI and post-LT HCC recurrence.

Our research has several limitations. First, IFLT is only carried out in a single center, and the overall sample size is insufficient. In the future, we will promote IFLT technology in multiple centers to increase the number of HCC patients to verify the significance of IFLT in reducing postoperative tumor recurrence. Second, there were not enough cases to perform stratified studies for different tumor stages. We will increase the number of HCC patients at different stages to explore the significance of IFLT in HCC patients within the standard or beyond the standard. Third, IFLT technology is currently limited to DBD donors, and we will explore the implementation of IFLT on DCD donors in the future. At that time, whether IFLT can reduce the recurrence rate of tumors after DCD donor liver transplantation still needs further research.

In conclusion, clinically, IRI increases the recurrence rate of HCC after liver transplantation. IFLT can significantly reduce IRI damage and has the potential to be a useful strategy to reduce HCC recurrence after liver transplantation.

\section{DATA AVAILABILITY STATEMENT}

The original contributions presented in the study are included in the article/supplementary materials, further inquiries can be directed to the corresponding author/s.

\section{ETHICS STATEMENT}

All the procedures were performed in accordance with the ethical standards of the responsible committee on human experimentation (institutional and national) and with the Helsinki Declaration of 1964 and later versions. The study was approved by the Institutional Ethics Committee for Clinical Research and Animal Trials of the First Affiliated Hospital of Sun Yat-sen University and informed consent waiver was granted by the IEC given the retrospective, minimal risk nature of the study. No organs from executed prisoners were transplanted into any of the patients reported in this study.

\section{AUTHOR CONTRIBUTIONS}

YT and TW were responsible for writing the manuscript. FL, QiZ, ZC, and JG were responsible for data collection and statistics. WJ, QiaZ, DW, and MC were responsible for the revision of the manuscript. $\mathrm{ZG}$ and $\mathrm{XH}$ were responsible for the design of the project. All authors contributed to the article and approved the submitted version.

\section{FUNDING}

Supported by grants as follows: the National Natural Science Foundation of China $(81970564,81471583,81570587$ and 81770410), Guangdong Basic and Applied Basic Research Foundation (2020A1515011557), the Key Clinical Specialty Construction Project of National Health and Family Planning Commission of the People's Republic of China, the Guangdong Provincial Key Laboratory Construction Projection on Organ Donation and Transplant Immunology (2013A061401007, 2017B030314018), Guangdong Provincial international Cooperation Base of Science and Technology (Organ Transplantation) (2015B050501002), Guangdong Provincial 
Natural Science Funds for Major Basic Science Culture Project (2015A030308010), Guangdong Provincial Natural Science Funds for Distinguished Young Scholars (2015A030306025), Special support program for training high level talents in Guangdong Province (2015TQ01R168), Pearl River Nova Program of Guangzhou (201506010014), Science and Technology Program of Guangzhou (201704020150), Sun Yatsen University Young Teacher Key Cultivate Project (17ykzd29)

\section{REFERENCES}

1. Bray F, Ferlay J, Soerjomataram I, Siegel R, Torre L, Jemal A. Global Cancer Statistics 2018: GLOBOCAN Estimates of Incidence and Mortality Worldwide for 36 Cancers in 185 Countries. CA: Cancer J Clin (2018) 68 (6):394-424. doi: 10.3322/caac.21492

2. Song TJ, Ip EW, Fong Y. Hepatocellular Carcinoma: Current Surgical Management. Gastroenterology (2004) 127(5 Suppl 1):S248-60. doi: 10.1053/ j.gastro.2004.09.039

3. Citores MJ, Lucena JL, de la Fuente S, Cuervas-Mons V. Serum Biomarkers and Risk of Hepatocellular Carcinoma Recurrence After Liver Transplantation. World J Hepatol (2019) 11(1):50-64. doi: 10.4254/ wih.v11.11.50

4. Agopian VG, Harlander-Locke M, Zarrinpar A, Kaldas FM, Farmer DG, Yersiz H, et al. A Novel Prognostic Nomogram Accurately Predicts Hepatocellular Carcinoma Recurrence After Liver Transplantation: Analysis of 865 Consecutive Liver Transplant Recipients. J Am Coll Surg (2015) 220 (4):416-27. doi: 10.1016/j.jamcollsurg.2014.12.025

5. Kornberg A, Küpper B, Tannapfel A, Katenkamp K, Thrum K, Habrecht O, et al. Long-Term Survival After Recurrent Hepatocellular Carcinoma in Liver Transplant Patients: Clinical Patterns and Outcome Variables. Eur J Surg Oncol (2010) 36(3):275-80. doi: 10.1016/j.ejso.2009.10.001

6. Orci LA, Lacotte S, Delaune V, Slits F, Oldani G, Lazarevic V, et al. Effects of the Gut-Liver Axis on Ischaemia-Mediated Hepatocellular Carcinoma Recurrence in the Mouse Liver. J Hepatol (2018) 68(5):978-85. doi: 10.1016/j.jhep.2017.12.025

7. Nagai S, Yoshida A, Facciuto M, Moonka D, Abouljoud MS, Schwartz ME, et al. Ischemia Time Impacts Recurrence of Hepatocellular Carcinoma After Liver Transplantation. Hepatology (2015) 61(3):895-904. doi: 10.1002/hep.27358

8. Kornberg A, Witt U, Kornberg J, Friess H, Thrum K. Extended Ischemia Times Promote Risk of HCC Recurrence in Liver Transplant Patients. Dig Dis Sci (2015) 60(9):2832-9. doi: 10.1007/s10620-015-3541-z

9. Huang H, Tohme S, Al-Khafaji AB, Tai S, Loughran P, Chen L, et al. DamageAssociated Molecular Pattern-Activated Neutrophil Extracellular Trap Exacerbates Sterile Inflammatory Liver Injury. Hepatology (2015) 62 (2):600-14. doi: 10.1002/hep.27841

10. Man K, Ng KT, Lo CM, Ho JW, Sun BS, Sun CK, et al. Ischemia-Reperfusion of Small Liver Remnant Promotes Liver Tumor Growth and MetastasesActivation of Cell Invasion and Migration Pathways. Liver Transpl (2007) 13 (12):1669-77. doi: 10.1002/lt.21193

11. Oldani G, Crowe LA, Orci LA, Slits F, Rubbia-Brandt L, de Vito C, et al. PreRetrieval Reperfusion Decreases Cancer Recurrence After Rat Ischemic Liver Graft Transplantation. J Hepatol (2014) 61(2):278-85. doi: 10.1016/j.jhep. 2014.03.036

12. Li CX, Ling CC, Shao Y, Xu A, Li XC, Ng KT, et al. CXCL10/CXCR3 Signaling Mobilized-Regulatory T Cells Promote Liver Tumor Recurrence After Transplantation. J Hepatol (2016) 65(5):944-52. doi: 10.1016/j.jhep. 2016.05.032

13. Yang F, Zhang Y, Ren H, Wang J, Shang L, Liu Y, et al. Ischemia Reperfusion Injury Promotes Recurrence of Hepatocellular Carcinoma in Fatty Liver via ALOX12-12hete-GPR31 Signaling Axis. J Exp Clin Cancer Res CR (2019) 38 (1):489. doi: 10.1186/s13046-019-1480-9

14. He XS, Guo ZY, Zhao Q, Ju WQ, Wang DP, Wu LW, et al. The First Case of Ischemia-Free Organ Transplantation in Humans: A Proof of Concept. Am J Transplant (2018) 18(3):737-44. doi: 10.1111/ajt.14583 and "Elite program" specially supported by China organ transplantation development foundation.

\section{ACKNOWLEDGMENTS}

Highly appreciate professor Shunjun Fu team for providing guidance in statistical analysis in this article.

15. Zhang Z, Tang Y, Zhao Q, Wang L, Zhu C, Ju W, et al. Association of Perfusion Characteristics and Posttransplant Liver Function in Ischemia-Free Liver Transplantation. Liver Transpl (2020) 26(11):1441-54. doi: 10.1002/ lt. 25825

16. Olthoff KM, Kulik L, Samstein B, Kaminski M, Abecassis M, Emond J, et al. Validation of a Current Definition of Early Allograft Dysfunction in Liver Transplant Recipients and Analysis of Risk Factors. Liver Transpl (2010) 16 (8):943-9. doi: 10.1002/lt.22091

17. Zhai Y, Petrowsky H, Hong J, Busuttil R, Kupiec-Weglinski J. IschaemiaReperfusion Injury in Liver Transplantation-From Bench to Bedside. Nat Rev Gastroenterol Hepatol (2013) 10(2):79-89. doi: 10.1038/nrgastro.2012.225

18. Souidi N, Stolk M, Seifert M. Ischemia-Reperfusion Injury: Beneficial Effects of Mesenchymal Stromal Cells. Curr Opin Organ Transplant (2013) 18(1):3443. doi: 10.1097/MOT.0b013e32835c2a05

19. Nasralla D, Coussios CC, Mergental H, Akhtar MZ, Butler AJ, Ceresa CDL, et al. A Randomized Trial of Normothermic Preservation in Liver Transplantation. Nature (2018) 557(7703):50-6. doi: 10.1038/s41586-018-0047-9

20. Reddy S, Greenwood J, Maniakin N, Bhattacharjya S, Zilvetti M, Brockmann J, et al. Non-Heart-Beating Donor Porcine Livers: The Adverse Effect of Cooling. Liver Transpl (2005) 11(1):35-8. doi: 10.1002/lt.20287

21. Eisenbach C, Encke J, Merle U, Gotthardt D, Weiss KH, Schneider L, et al. An Early Increase in Gamma Glutamyltranspeptidase and Low Aspartate Aminotransferase Peak Values are Associated With Superior Outcomes After Orthotopic Liver Transplantation. Transplant Proc (2009) 41(5):172730. doi: 10.1016/j.transproceed.2009.01.084

22. Wadei HM, Lee DD, Croome KP, Mai ML, Golan E, Brotman R, et al. Early Allograft Dysfunction After Liver Transplantation Is Associated With Shortand Long-Term Kidney Function Impairment. Am J Transplant (2016) 16 (3):850-9. doi: 10.1111/ajt.13527

23. Deschenes M. Early Allograft Dysfunction: Causes, Recognition, and Management. Liver Transpl (2013) 19 Suppl 2:S6-8. doi: 10.1002/lt.23746

24. Mazzaferro V, Llovet JM, Miceli R, Bhoori S, Schiavo M, Mariani L, et al. Predicting Survival After Liver Transplantation in Patients With Hepatocellular Carcinoma Beyond the Milan Criteria: A Retrospective, Exploratory Analysis. Lancet Oncol (2009) 10(1):35-43. doi: 10.1016/s14702045(08)70284-5

25. Gouw AS, Balabaud C, Kusano H, Todo S, Ichida T, Kojiro M. Markers for Microvascular Invasion in Hepatocellular Carcinoma: Where do We Stand? Liver Transpl (2011) 17 Suppl 2:S72-80. doi: 10.1002/lt.22368

26. Berry K, Ioannou GN. Serum Alpha-Fetoprotein Level Independently Predicts Posttransplant Survival in Patients With Hepatocellular Carcinoma. Liver Transpl (2013) 19(6):634-45. doi: 10.1002/lt.23652

27. Hameed B, Mehta N, Sapisochin G, Roberts JP, Yao FY. Alpha-Fetoprotein Level $>1000 \mathrm{Ng} / \mathrm{mL}$ as an Exclusion Criterion for Liver Transplantation in Patients With Hepatocellular Carcinoma Meeting the Milan Criteria. Liver Transpl (2014) 20(8):945-51. doi: 10.1002/lt.23904

28. Jassem W, Xystrakis E, Ghnewa YG, Yuksel M, Pop O, Martinez-Llordella M, et al. Normothermic Machine Perfusion (NMP) Inhibits Proinflammatory Responses in the Liver and Promotes Regeneration. Hepatology (2019) 70 (2):682-95. doi: 10.1002/hep.30475

29. Ling Q, Liu J, Zhuo J, Zhuang R, Huang H, He X, et al. Development of Models to Predict Early Post-Transplant Recurrence of Hepatocellular Carcinoma That Also Integrate the Quality and Characteristics of the Liver Graft: A National Registry Study in China. Surgery (2018) 16(8):1-10. doi: 10.1016/j.surg.2018.01.022 
30. Kulik LM, Fisher RA, Rodrigo DR, Brown RSJr., Freise CE, Shaked A, et al. Outcomes of Living and Deceased Donor Liver Transplant Recipients With Hepatocellular Carcinoma: Results of the A2ALL Cohort. Am J Transplant (2012) 12(11):2997-3007. doi: 10.1111/j.1600-6143.2012.04272.x

31. Man K, Fan ST, Lo CM, Liu CL, Fung PC, Liang TB, et al. Graft Injury in Relation to Graft Size in Right Lobe Live Donor Liver Transplantation: A Study of Hepatic Sinusoidal Injury in Correlation With Portal Hemodynamics and Intragraft Gene Expression. Ann Surg (2003) 237(2):256-64. doi: 10.1097/ 01.Sla.0000048976.11824.67

32. Mueller M, Kalisvaart M, O'Rourke J, Shetty S, Parente A, Muller X, et al. Hypothermic Oxygenated Liver Perfusion (HOPE) Prevents Tumor Recurrence in Liver Transplantation From Donation After Circulatory Death. Ann Surg (2020) 272(5):759-65. doi: 10.1097/sla.000000000 0004258

33. Xu J, Sayed BA, Casas-Ferreira AM, Srinivasan P, Heaton N, Rela M, et al. The Impact of Ischemia/Reperfusion Injury on Liver Allografts From Deceased After Cardiac Death Versus Deceased After Brain Death Donors. PloS One (2016) 11(2):e0148815. doi: 10.1371/journal.pone.0148815

34. Huang C, Chen Z, Wang T, He X, Chen M, Ju W. A Marginal Liver Graft With Hyperbilirubinemia Transplanted Successfully by Ischemia-Free
Liver Transplantation. Ann Transl Med (2021) 9(5):425. doi: 10.21037/ atm-20-6296

Conflict of Interest: The authors declare that the research was conducted in the absence of any commercial or financial relationships that could be construed as a potential conflict of interest.

Publisher's Note: All claims expressed in this article are solely those of the authors and do not necessarily represent those of their affiliated organizations, or those of the publisher, the editors and the reviewers. Any product that may be evaluated in this article, or claim that may be made by its manufacturer, is not guaranteed or endorsed by the publisher.

Copyright (c) 2021 Tang, Wang, Ju, Li, Zhang, Chen, Gong, Zhao, Wang, Chen, Guo and He. This is an open-access article distributed under the terms of the Creative Commons Attribution License (CC BY). The use, distribution or reproduction in other forums is permitted, provided the original author(s) and the copyright owner(s) are credited and that the original publication in this journal is cited, in accordance with accepted academic practice. No use, distribution or reproduction is permitted which does not comply with these terms. 